\title{
LES RÉSIDUS DE PESTICIDES DANS LE LAIT ET LES PRODUITS LAITIERS (1)
}

\author{
par \\ Gustave THIEULIN, Jean PANTALÉN, Lueien RICHOU \\ et Guy CUMONT
}

Destinés à lutter contre les vecteurs de maladies humaines ou animales, et contre les ennemis des plantes ou des récoltes, les pesticides ont pris une importance majeure dans le monde moderne.

Sans eux, certaines contrées demeureraient difficilement habitables et nombre de denrées alimentaires ne pourraient être produites économiquement et parfois même plus produites du tout. Indiquons par exemple, que d'après de récentes estimations d'organismes internationaux (O.M.S.-F.A.O.), leur suppression entraînerait, aux Etats-Unis, une diminution de 50 p. 100 en ce qui concerne la production des pommes de terre, des fruits et du coton, et d'au moins 25 p. 100 en ce qui concerne la viande, la laine et le lait. Cette nécessité de posséder des produits doués d'une très grande activité antiparasitaire est d'autant plus évidente, que plus des deux tiers de la population totale du globe sont encore sousalimentés.

Mais l'intensification de l'emploi de ces agents chimiques, naturels ou de synthèse, particulièrement actifs, comporte des risques de nocivité indéniable (Le Moan [4]). Dans la très grande majorité des cas, les pesticides organiques de synthèse, par exemple, exercent leur action toxique non seulement sur les parasites à détruire mais encore sur de nombreux insectes utiles, sur le gibier, les animaux domestiques et finalement risquent d'atteindre l'homme par la voie des résidus absorbés avec les aliments. Ainsi se trouve posé le problème d'une toxicité à long terme.

Les insecticides organo-chlorés, à ce point de vue, paraissent les plus redoutables; leur stabilité chimique et leur grande liposolubilité les font persister et s'accumuler dans un très grand nombre de denrées d'origine végétale ou animale.

Parmi celles-ci, le lait et les produits laitiers, en raison de leur teneur élevée en matière grasse, paraissent les plus exposés. C'est

(1) Bull. A cad. nat. méd., 1966, 150, nos 24-25, 478. Recherches effectuées dans les laboratoires du Service vétérinaire sanitaire de Paris et du département de la Seine (Rapports d'activité 1964 et 1965). 
pour cette raison que parmi les denrées d'origine animale, nous nous sommes attachés, depuis le début de l'année 1964, à la recherche de résidus de pesticides à partir d'échantillons de laits, de beurres et de fromages provenant de régions de technicités agricoles différentes.

\section{Pesticides recherchés}

Pour les raisons que nous venons de souligner, ce sont les insecticides organo-chlorés, qui retiennent avant tout l'attention des hygiénistes. Nous avons particulièrement recherché (tableau I) :

- L'HCH : 1-2-3-4-5-6 hexachlorocyclohexane sous ses trois isomères principaux $\alpha, \beta$ et $\gamma$ (lindane) ;

- La dieldrine: 1-2-3-4-10-10 hexachloro 6-7 époxy-1-4-4a5-6-7-8-8a octahydro-1-4-5--8 diméthanonaphtalène; il s'agit du métabolite époxyde naturel de l'adrénaline ou 1-2-3-4-10-10 hexachloro 1-4-4a-5-8-8a hexahydro 1-4-5-8- diméthanonaphthalène ;

- L'heptachlore époxyde ou 1 (ou 3a) 4-5-6-7-8-8 heptachloro 2-3 époxy-2-3-3a-4-7-7a hexahydro-4, 7 méthano indène, qui est le métabolite époxyde naturel de l'heptachlore ou heptachloro tétrahydro méthano indène ;

- Le D.D.T. ou 1-1-1 trichloro-2-2bis parachlorophényléthane. Dans ses deux isomères $0 . p^{\prime}$ et p.p' et son dérivé métabolite le D.D.E. 2-2bis parachlorophényl 1-1 dichloréthylène.

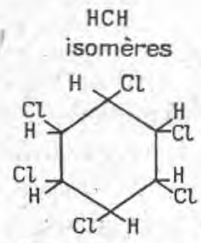

1.2.3.4.5.6 héxachloro cyclohéxane

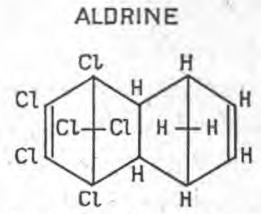

1.2.3.4.10.10 héxachloro

1.4.4. a5.8.8 a héxahydro 1458 diméthano naphtalène
DIELDRINE

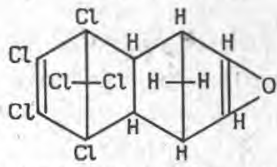

1.2.3.4.10.10 héxachloro 6.7.epoxy

1.4.4 a 5.6.7.8.8.a octahydro

1458 diméthano naphtalène

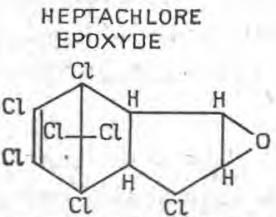

1.4.5.6.7.8.8 héptachloro 23 epoxy 2.3.3a 4.7.7a hexa hydro 47 méthano indène
DDT

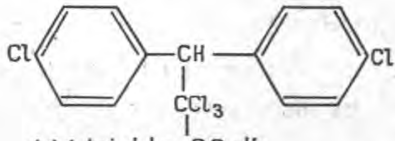

1.1.1 trichlo $22 \mathrm{di}$ parachlorophényléthane
DDE

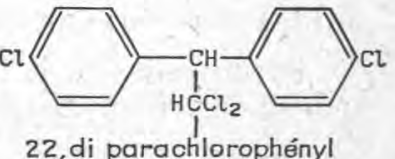

11 dichlorèthylène

TABLEAU I 
Tous ces produits, notamment l'HCH, ont la faculté de persister plusieurs années dans les sols. Certains d'entre eux, l'aldrine, l'heptachlore, sont ou ont été utilisés mélangés à des engrais (cas de la région $\mathrm{A}$ ), au taux de $4 \mathrm{~kg}$ de produit pur à l'hectare. Leur emploi est également devenu courant dans la désinsectisation des étables et des locaux d'habitation.

Bien entendu, se pose aussi le problème des insecticides organophosphorés. Mais étant donné les conditions particulières de leur métabolisation, ils sont essentiellement des agents d'intoxication aiguë chez l'animal et dépourvus pratiquement du pouvoir de s'accumuler à l'état de résidu dans leurs tissus et dans le lait.

\section{Méthode analytique}

Dans son principe, elle repose sur deux étapes chromatographiques. Le produit expertisé abandonne tout d'abord, d'une manière sélective, ses résidus organo-chlorés, par passage sur une colonne chromatographique de silice. Cet extrait purifié est ensuite élué par chromatographie en phase gazeuse. Le résultat apparaît sur un chromatogramme sous forme de pics. Le temps d'émergence de chaque pic est la caractéristique qualitative de la substance en cause ; leur surface permet de préciser les données quantitatives, la limite de sensibilité globale de l'analyse étant de l'ordre de $0,01 \mathrm{ppm}$ (tableau II).

\section{1) Extraction et purification.}

Langlois, Stemp et Liska d'une part [3] et Moats d'autre part [6], ont mis au point dès 1963 une méthode efficace d'isolement que nous avons adoptée et très légèrement modifiée au cours de nos recherches systématiques.

L'extraction se fait par un mélange dichlorométhane/éther de pétrole; la purification est réalisée par chromatographie sur colonne à l'aide d'absorbant sélectif (Florisil).

\section{2) Dosage.}

Il s'effectue par chromatographie en phase gazeuse, à l'aide d'un détecteur à capture d'électrons (type Lovelock-Lipsky [5]), après passage sur différentes eolonnes de séparation (Dow 11 , Qf 1, Carbowax $20 \mathrm{M})$.

La méthode de partition hexane/acétonitrile et l'oxydation sulfochromique de l'échantillon ont été expérimentées comme complément d'identification. Toutes les solutions standards de référence nous ont été obligeamment fournies par les Services du Laboratoire de Phyto-Pharmacie de l'I.N.R.A. à Versailles, 

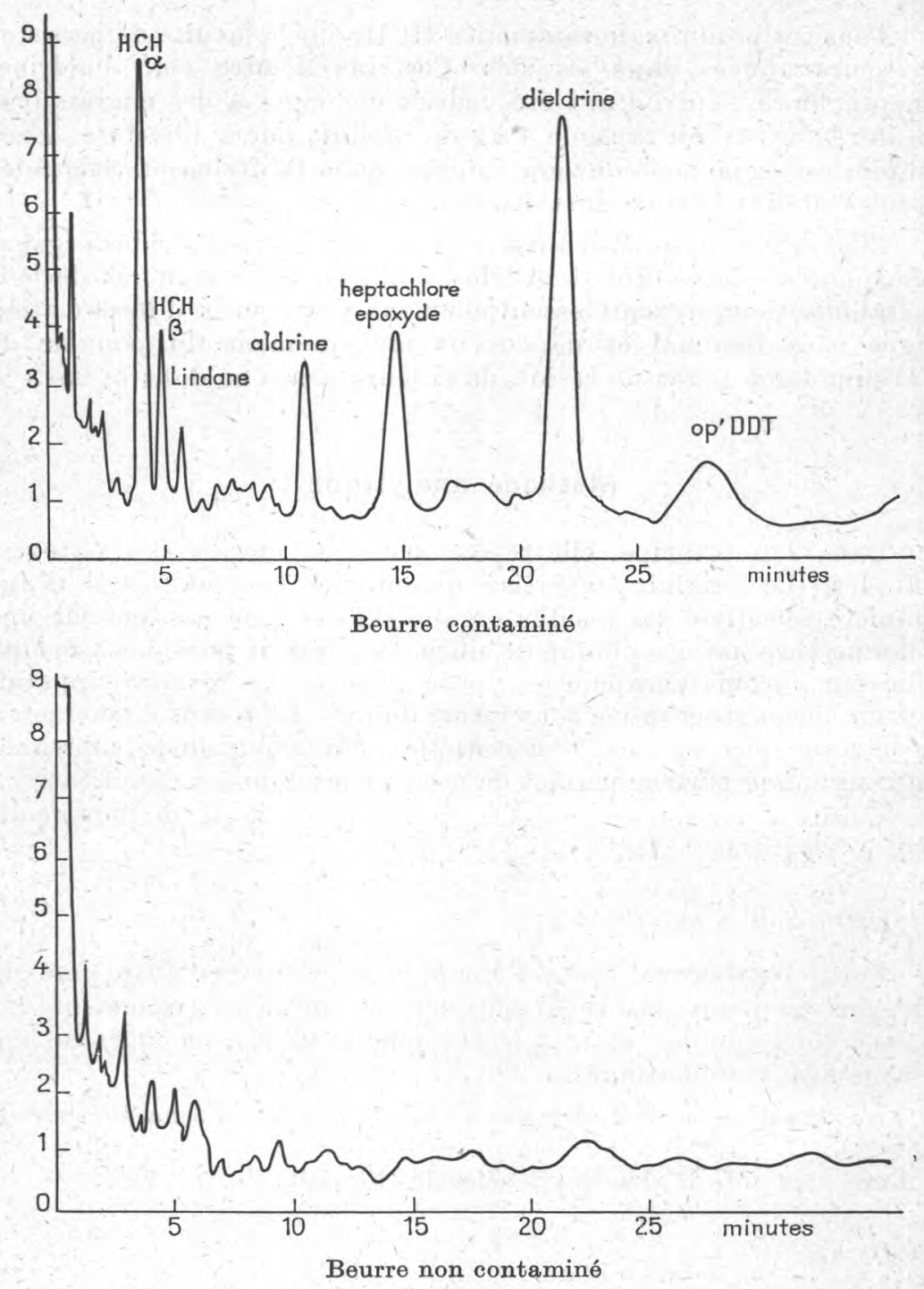

TABLEAU II

Services dirigés par MM. Viel [8] et Hascoet que nous tenons spécialement à remercier.

De plus, des essais ont été réalisés sur des beurres témoins que nous avons nous-mêmes préparés à partir de laits provenant d'une région de montagne (Pyrénées) très localisée ayant échappé jusqu'à présent à tout traitement par insecticide organo-chloré. 


\section{Résultats}

Le tableau récapitulatif ci-après, rassemble les résultats acquis durant les deux années 1964 et 1965 à la suite de l'analyse de 225 échantillons se décomposant comme suit : laits, 115 ; beurres, 78 ; fromages, 32.

Ne figurent pas sur ce tableau les données relatives au D.D.T. et à l'heptachlore époxyde, substances qui n'ont jamais été trouvées en quantités dosables mais seulement à l'état de traces dans certains échantillons. Par contre, tous les autres pesticides ont pu être dosés avec régularité (tableau III).

Si nous bloquons les résultats des deux années 1964 et 1965 et ne retenons que les échantillons jugés positifs, le bilan, concernant les résidus totalisés, est alors le suivant :

- 74 p. 100 des laits crus sont contaminés à des taux globaux variant de 0,07 à $0,15 \mathrm{ppm}$;

- 92 p. 100 des beurres de la région $\mathrm{A}$ sont pollués à des taux globaux variant de 1,85 à $3,45 \mathrm{ppm}$ (facteur de multiplication de l'ordre de 20 à 25 par rapport au lait);

- 87 p. 100 des beurres de la région $B$ sont pollués, mais à des taux globaux plus faibles 1,50 à. $1,80 \mathrm{ppm}$;

- 60 p. 100 des fromages, tous issus de la région $A$, ont des taux globaux de résidus oscillant de 0,50 à $0,70 \mathrm{ppm}$.

\section{Commentaires}

La présence de résidus d'insecticides organo-chlorés dans les laits et les produits laitiers se manifeste dans 60 à 92 p. 100 des échantillons examinés; ce pourcentage, aussi surprenant qu'il paraisse, est la conséquence naturelle de l'emploi constant de ces produits chimiques en agriculture durant ees vingt dernières années.

Bien que les doses de chaque pesticide pris en particulier paraissent faibles (mis à part le taux 1,20 $\mathrm{mg}$ de résidu de dieldrine trouvé en 1964 par kilogramme de beurre), les taux globaux maxima atteints, avec certaines provenances, ont été de $0,15 \mathrm{mg} / \mathrm{l}$ de lait et de $3,45 \mathrm{mg} / \mathrm{kg}$ de beurre.

Les taux de résidus sont d'ailleurs plus forts, dans l'ensemble, en 1964 : influence climatique - année de sécheresse - emploi de produits inutilisés ultérieurement. En 1965 (et au début 1966), une légère baisse des taux d'HCH notamment, s'est accentuée dans les produits originaires de la région $\mathrm{A}$, région qui a été particulièrement étudiée. Les taux de l'isomère $\gamma$ (lindane) ont, dans l'en- 


\section{TABLEAU III}

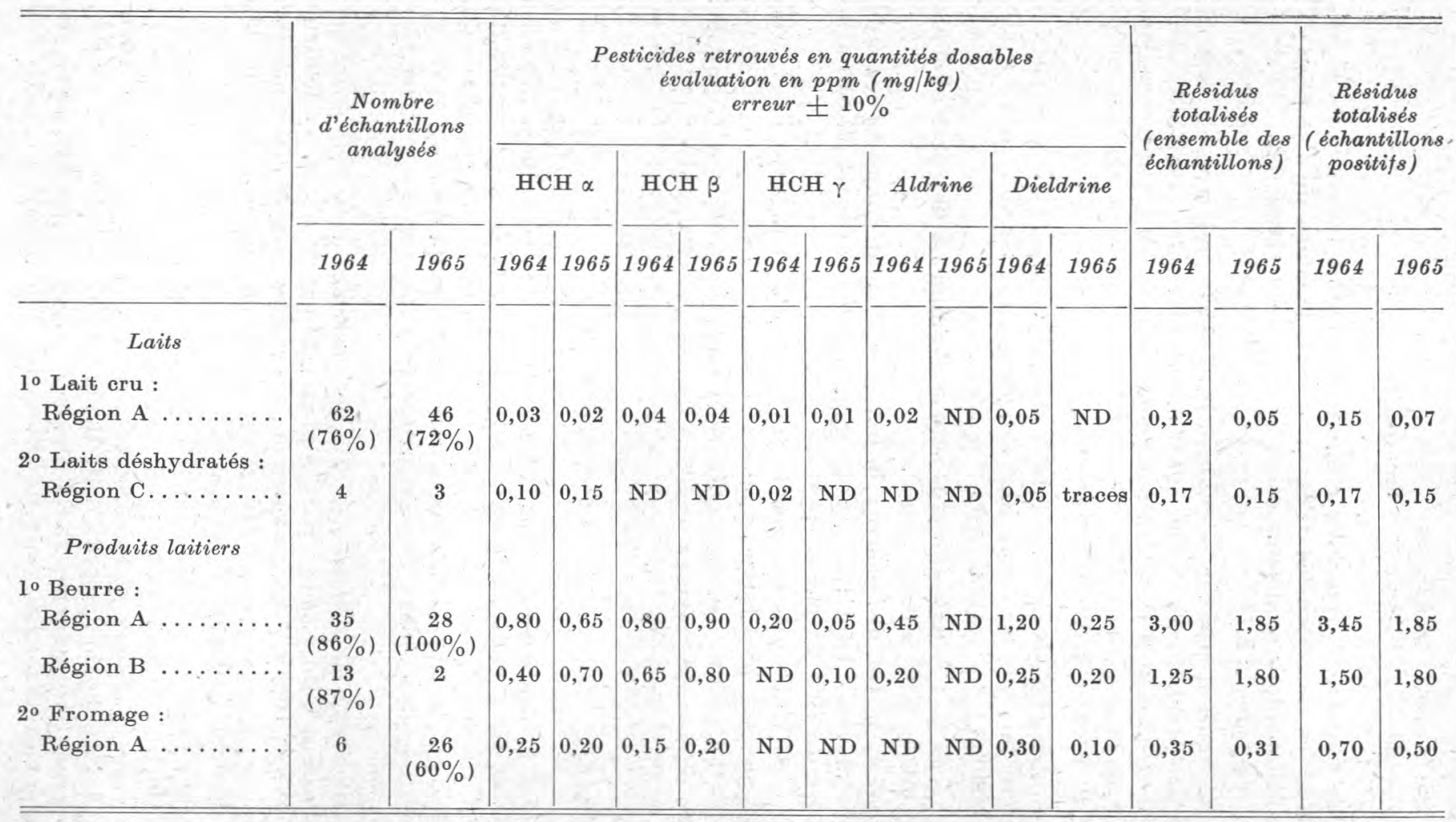

Les indications portées entre parenthèses indiquent le pourcentage des échantillons où les résidus ont été trouvés en quantités dosables. ND : pesticide non décelable. 
semble, toujours été assez faibles (extraction plus difficile, produit métabolisé par l'organisme animal). L'aldrine n'a plus été décelée (en quantité dosable) et le taux résiduel de la dieldrine est tombé de $1,17 \mathrm{ppm}$ en 1964 à $0,25 \mathrm{ppm}$ en 1965 . Cet insecticide n'est plus trouvé qu'à l'état de traces depuis le deuxième semestre 1965 . Ceci pourrait être consécutif à l'abandon progressif de l'aldrine autrefois utilisé pour l'enrobage des graines de semence et interdite par décision ministérielle du ler octobre 1962.

Ces résultats concordent par ailleurs avec des analyses régulières effectuées sur du gibier, depuis 1962, par les Laboratoires de l'I.N.R.A.

De toute façon, quelles que soient les variations inéluctables d'une année sur l'autre (tableau III, les taux maxima atteints doivent retenir l'attention. M. le professeur Truhaut [7] insiste, avec juste raison, depuis de nombreuses années, sur les risques de toxicité à long terme, pouvant résulter des effets cumulatifs des pesticides absorbés avec les aliments.

\section{Règles de sécurité}

Les préventions contre de tels risques se fondent, à l'étranger, dans de nombreux cas, sur l'existence de Normes concernant les doses maxima tolérables dans les aliments (Etats-Unis, Canada, Russie et récemment Hollande). La politique américaine (Food and Drug Administration) est de considérer tout résidu trouvé dans le lait comme un agent d'adultération (Henderson [2]). Cependant, en raison des limites de sensibilité des méthodes d'analyse officielle de référence, il est appliqué actuellement le "zéro administratif " correspondant à $2,5 \mathrm{ppm}$ de résidus de pesticides rapportés à la matière grasse.

Ce principe n'est pas en vigueur en France où sont seulement édictées une liste positive de pesticides autorisés et une interdiction d'emploi dans des délais fixes avant la récolte des végétaux.

Ce système, très valable en lui-même, nécessite un contrôle très strict et des vérifications très nombreuses, sans rapport avec ce qui est possible dans la réalité. De plus, si on se réfère aux dispositions légales actuelles, les aliments ne devraient contenir aucun résidu : seuls sont réglementés le lindane, le malathion et les pyréthrines en tant que substances tolérées dans certains produits alimentaires végétaux.

Tenant compte des nécessités économiques de l'agriculture moderne et de l'impossibilité de supprimer radicalement l'emploi de nombreux pesticides, la politique réaliste dicte d'appliquer désormais en France une limite tolérable de résidus de pesticides pour chaque denrée. 
Les étudès que nous venons d'effectuer sur le lait et les produits laitiers nous permettent de proposer actuellement pour limites maxima tolérables des résidus totalisés : $0,10 \mathrm{ppm}$ rapporté au lait total et 2,50 ppm rapporté à la matière grasse.

\section{Conclusion}

Les recherches effectuées en deux ans sur 225 échantillons de lait et de produits laitiers provenant de deux grandes régions agricoles françaises, ont révélé, dans la majorité des cas, une contamination par des résidus de pesticides organo-chlorés.

Ces résidus retrouvés dans une proportion variant de 60 à 92 p. 100 selon les types d'échantillon, sont constitués, essentiellement par de l'HCH et de la dieldrine; le niveau maximum de résidus totalisés ayant été de $0,15 \mathrm{mg} / \mathrm{l}$ de lait, de $3,45 \mathrm{mg} / \mathrm{kg}$ de beurre et $0,70 \mathrm{mg} / \mathrm{kg}$ de fromage.

Ces données mettent en évidence l'insuffisance des méthodes prophylactiques actuellement en vigueur. Elles posent la nécessité de contrôles analytiques systématiques et l'établissement de doses limites tolérables pour les résidus de pesticides dans les aliments.

\section{BIBLIOGRAPHIE}

[1] F.A.O.-O.M.S. Principes devant régir la protection de la santé des consommateurs à l'égard des résidus de pesticides. Rapport technique $\mathrm{n}^{0} 240,1962$. Evaluations of the toxicity of pesticide residues in food. F.A.O. Meeting, Report no PL/1965/10/1, WHO/FOOD Add/27.65.

[2] HENDERSON (J. L.). Insecticide residues in milk and dairy produets. Res. Rev., 1965, 8, 74.

[3] Langlois (B. E.), Stemp (A. R.) and Liska (B. J.). Rapid cleanup of dairy products for analysis of chlorinated insecticide residue by electron capture gas chromatography. $J$. of Agric. and Food chem., $1964,12,243$.

[4] Le Moan (G.). Intoxication par les pesticides. Etude chimique et toxicologique. Arch. Mal, prof., 1961, 22, 226.

[5] Lovelock (J. E.) and Lipsky (S. R.). Electron affinity spectroscopy. A new method for the identification of functional groups in chemical compounds separated by gas ehromatography. $J$, Amer. chem. Soc., 1960, 82, 431.

[6] Moats (W. A.). One step chromatographic clean up of chlorinated. hydrocarbon pesticide residues in butterfat. II. Chromatography on florisil. J. of Assoc. Off. Agr. chem., 1963, 46, 172.

[7] Truhaut (R.). Aperçu sur les risques de nocivité pouvant résulter de la présence de pesticides dans les aliments. Méthodes de prévention. C. R. III e Symposium de l'I.J.A., mai 1957, Côme.

[8] Truhaut (R.) et Viel (G.). Lutte antiparasitaire et toxicité. Bull. tech. Inf. Ing. Serv, Agr., 1962, 167, 160. 